\title{
Relative Change in NT-proBNP Level: An Important Risk Predictor of Cardiovascular Congestion in Haemodialysis Patients
}
M. Pastural-Thaunat ${ }^{\mathrm{a}}$
R. Ecochard ${ }^{b, d}$
N. Boumendjel ${ }^{\mathrm{a}}$
E. Abdullah ${ }^{a}$
C. Cardozo ${ }^{\mathrm{a}}$
A. Lenz $z^{a}$
I. $\mathrm{M}^{\prime} \mathrm{Pio}^{\mathrm{a}}$
J.C. Szelag ${ }^{\mathrm{a}}$
D. Fouque ${ }^{C}$
A. Walid ${ }^{a}$
M. Laville ${ }^{\mathrm{a}, \mathrm{c}}$

\begin{abstract}
${ }^{a}$ Association pour I'Utilisation du Rein Artificiel à Lyon, ${ }^{b}$ Service de Biostatistique, Hospices Civils de Lyon, and 'Pôle de Médecine, Hôpital Edouard Herriot, Lyon, and ' Laboratoire de Biométrie et Biologie Evolutive, CNRS UMR-5558, Université Lyon 1, Villeurbanne, France
\end{abstract}

\section{Key Words}

Congestive heart failure $\cdot$ Haemodialysis $\cdot$ NT-proBNP $\cdot$ Monitoring $\cdot$ Risk predictor

\begin{abstract}
Background: Cross-sectional studies have shown that B-type natriuretic peptide (BNP) and its $\mathrm{N}$-terminal fragment (NT-proBNP) are predictive of cardiovascular death in haemodialysis (HD) patients. In the present study, we tested the hypothesis that monitoring NT-proBNP measurements adds further prognostic information, i.e. predicts congestive heart failure (CHF) events. Methods: In a prospective cohort of 236 HD patients, NT-proBNP levels were measured monthly during 18 months. Patients were divided according to the occurrence of CHF events. In a nested case-control study, we assessed the evolution of NT-proBNP levels. Results: On average, the $236 \mathrm{HD}$ patients were followed up for 12.5 months, a period during which 44 patients developed a CHF event (half requiring hospitalisation). At baseline, patients who developed a CHF event had significantly more dilated cardiomyopathy and/or altered left ventricular ejection fraction and higher NT-proBNP levels compared with patients who did not develop a CHF event. During follow-up, we observed a significant increase in NT-proBNP levels preceding the CHF event. At a $20 \%$ relative increase of NT-proBNP, the sensitivity of NT-proBNP as a predictor of CHF events was 0.57 and the specificity 0.77 . Conclusion: The relative change in NT-proBNP levels is a significant risk predictor of a CHF event.

Copyright $\odot 2012$ S. Karger AG, Basel
\end{abstract}

The results presented in this paper have not been published previously in whole or part, except in abstract format.

Dr. Myriam Pastural-Thaunat

AURAL

124 rue Villon

FR-69008 Lyon (France)

E-Mail myriam.pastural@auralyon.com 


\section{Introduction}

The annual mortality rate among chronic haemodialysis (HD) patients approaches $20 \%$. Approximately half of the deaths are attributable to cardiovascular causes. What is less appreciated is that, according to the US Renal Data System, 217 hospitalisations per 1,000 patient-years are attributed to congestive heart failure (CHF) [1].

In the general population, single measurements of brain natriuretic peptide (BNP) and its N-terminal fragment (NT-proBNP) accurately discriminate between $\mathrm{CHF}$ and other causes of breathlessness that lead to hospital admissions [2].

Concentrations of these natriuretic peptides are related to left ventricular (LV) filling pressures and wall stress [3]. In dialysis patients, fluid overload and LV structural and functional abnormalities are both frequent and difficult to differentiate. In dialysis patients, BNP and NT-proBNP levels are almost invariably increased, and the 'normal' range in this population is not defined [4].

In asymptomatic HD patients, single, cross-sectional measurements of NT-proBNP are powerful predictors for all-cause mortality and cardiovascular death, and baseline NTproBNP measurements may facilitate efforts to stratify the risk in patients starting HD [5-8].

However, few studies have examined the diagnostic utility of serial assessments of NTproBNP in the HD population. Nevertheless, monitoring BNP levels is currently used for patients with heart failure as Troughton et al. [9] showed that BNP-guided treatment of heart failure reduced total cardiovascular events and delayed the time to the first event compared with intensive clinically guided treatment.

In the family of natriuretic peptides, NT-proBNP seems to be the best predictor of clinical outcome and the best marker of extracellular fluid overload. This peptide is larger and has a longer half-life than BNP (the active form), making its measurement easier [10].

Therefore, we sought to examine the relationship between an increase in NT-proBNP levels and future clinical events in a prospective cohort, i.e. whether follow-up NT-proBNP measurements add further prognostic information in HD patients. More specifically, we assessed the value of the relative changes in NT-proBNP as independent predictive markers of CHF events.

\section{Patients and Methods}

\section{Study Design and Study Population}

A prospective cohort study of HD patients was conducted during 18 months. Both prevalent and incident cases were included, with no exclusion criteria. A total of 236 patients were included in the study. The medical history of all patients was collected at baseline, and events occurring during follow-up were systematically recorded. Patients were followed up for the occurrence of medical adverse events, i.e. CHF events, cardiac ischaemia, arrhythmia, hospitalisation for other cardiac events or infectious disease, and death. The patients were censored at the time of renal transplantation, death, and first CHF event. This study was approved by the local institutional review board, and informed consent was obtained from all patients who participated in the study.

\section{Studied Variable: NT-proBNP}

At the beginning of the follow-up, i.e. at baseline, measurement of the NT-proBNP level was performed every month. Serum NT-proBNP was quantified by electrochemiluminescence immunoassay on the Cobas E601 analyser (Roche Diagnostics Corp.). The variability 
of the NT-proBNP assay is always below 6\% in our lab (recommended: below 9\%). NT-proBNP measurements after a CHF event were discarded.

Outcome: CHF Events

A CHF event was defined as the first admission for CHF requiring hospitalisation and any episode of CHF requiring a session of additional dialysis as an emergency in our centre. The clinical diagnosis of CHF was made by the attending physician on the basis of the presence of symptoms and signs of CHF including moderate to severe dyspnoea (class III and IV according to the New York Heart Association), raised jugular venous pressure, and basal crepitations. This information was retrieved from the computerized MEDIAL medical record system (ECHO, Nantes, France) that keeps detailed records of all hospitalisation and/ or acute events.

\section{Potential Confounding Factors}

The following characteristics of the patients were collected at baseline to adjust our analyses: age and sex, weight and height (body mass index), time on dialysis, comorbidities including diabetes, coronary artery disease, valvular heart disease, arrhythmia, hypertrophic cardiomyopathy, dilated cardiomyopathy, and altered LV ejection fraction (LVEF; $<40 \%$ on 2-dimensional echocardiography).

\section{Statistical Analysis}

The characteristics of the patients at baseline were tabulated. Quantitative variables were presented as mean \pm standard deviation (min-max) and binary variables were presented as proportions. Then, patients were divided according to the outcome criteria, i.e. the occurrence of a CHF event, and their characteristics and levels of NT-proBNP at baseline were compared. We assessed the evolution of NT-proBNP separately in patients without a CHF event and in patients who developed a CHF event. This regression analysis was a nested casecontrol study. In a nested case-control study, cases of a disease that occur in a defined cohort are identified and, for each, a specified number of matched controls is selected from among those in the cohort who have not developed the disease by the time of disease occurrence in the case. The nested case-control design is particularly advantageous for studies of biologic disease precursors.

The evolution of the NT-proBNP measurements before a CHF event was compared to the evolution of the NT-proBNP measurements at the same time for the controls, using a polynomial regression for repeated measurements. Then, the usefulness of the relative change in NT-proBNP level to predict the risk of a CHF event was assessed by the analysis of receiver operating characteristic (ROC) curves. The relative change in NT-proBNP was defined as the proportion of increase or decrease in NT-proBNP measured compared to the previous measurement.

All statistical analyses were made using Stata 10 Software. Tests were considered to be statistically significant when $\mathrm{p}<0.05$.

\section{Results}

\section{Patient Characteristics}

Our study cohort included $236 \mathrm{HD}$ patients. The mean age of the population was 59.84 \pm 17.4 years (18.3-88.3), and $36 \%$ were female. The mean HD duration was $46 \pm 74.3$ months (0-482). In total, $61.4 \%$ of our patients were Caucasian, $11.5 \%$ were Black, and $27.1 \%$ of other origins. Patients had a history of diabetes in $25 \%$ of the cases and a history of coro- 
Table 1. Baseline characteristics of patients with/without CHF event during the follow-up

\begin{tabular}{lccc}
\hline & $\begin{array}{l}\text { No CHF event } \\
(\mathrm{n}=192 ; 81.36 \%)\end{array}$ & $\begin{array}{l}\text { CHF event } \\
(\mathrm{n}=44 ; 18.64 \%)\end{array}$ & p value* \\
\hline Female sex & $66(34.4)$ & $19(43.2)$ & 0.27 \\
Age, years & $59.7 \pm 1.22$ & $59.98 \pm 2.88$ & 0.921 \\
Body mass index & $25.1 \pm 0.33$ & $21.95 \pm 0.84$ & 0.000 \\
Time on HD, months & $42.05 \pm 5.24$ & $63.28 \pm 12.11$ & 0.088 \\
Diabetes mellitus & $48(25)$ & $11(25)$ & 1.0 \\
Comorbid conditions & & $17(38.6)$ & 0.194 \\
$\quad$ Coronary artery disease & $55(28.6)$ & $3(6.8)$ & 0.99 \\
$\quad$ Valvular heart disease & $13(6.8)$ & $7(15.9)$ & 0.10 \\
Arrhythmia & $15(7.9)$ & $16(41)$ & 0.063 \\
Hypertrophic cardiomyopathy & $44(26)$ & $8(18.2)$ & 0.007 \\
$\quad$ Dilated cardiomyopathy & $9(4.7)$ & $21(47.7)$ & $<0.0001$ \\
$\quad$ Altered LVEF & $33(17.2)$ & 7,982 & $<0.0001$ \\
Baseline NT-proBNP, ng/l** & 2,438 &
\end{tabular}

Values are number of patients (\%) or means \pm SD.

* Pearson chi square. ${ }^{* *}$ Geometric mean.

nary artery disease in 30.5\%. Echocardiographic data from baseline showed hypertrophic cardiomyopathy, dilated cardiomyopathy, and altered LVEF in 25.4, 7.2, and 23\% of the cases, respectively.

\section{Outcomes}

The mean follow-up was 12.5 months (2-18), during which 44 (18.6\%) patients developed a CHF event, of which 22 patients required hospitalisation and 22 patients required a session of additional dialysis in our centre. The length of hospitalisation was $8.64 \pm 6.1$ days (1-21), depending on the need for further exploration or the patient's general condition. None of the patients needed mechanical ventilation.

Table 1 provides a comparison of the patients' baseline characteristics according to their evolution with or without a CHF event.

Baseline NT-proBNP levels were significantly higher among patients who subsequently developed a CHF event than among those who did not (2,438 vs. 7,982 ng/l, respectively). Patients who developed a CHF event had significantly more dilated cardiomyopathy (18.2 vs. $4.7 \%$, respectively) and/or altered LVEF ( 47.7 vs. $17.2 \%$, respectively) at baseline compared with patients who did not develop a CHF event. The body mass index was lower in the group of patients who subsequently developed a CHF event.

\section{Change in NT-proBNP as a Predictor of CHF Events}

The graphical representation of the evolution of NT-proBNP before a CHF event showed a clear increase of the biomarker during the months preceding the CHF event (fig. 1). The regression analysis showed that this difference of evolution was statistically significant ( $\mathrm{p}<$ 0.05 ) for the measurement during approximately the 2 months preceding the event.

\section{Relative Change in NT-proBNP as a Predictor for Clinical Practice}

The proportion of the increase or decrease in NT-proBNP measured at two consecutive visits was assessed as a predictor of a CHF event (fig. 2). The AUC was 0.80. If the threshold of positivity was chosen in favour of specificity, then, at $20 \%$ of the relative increase in NT- 


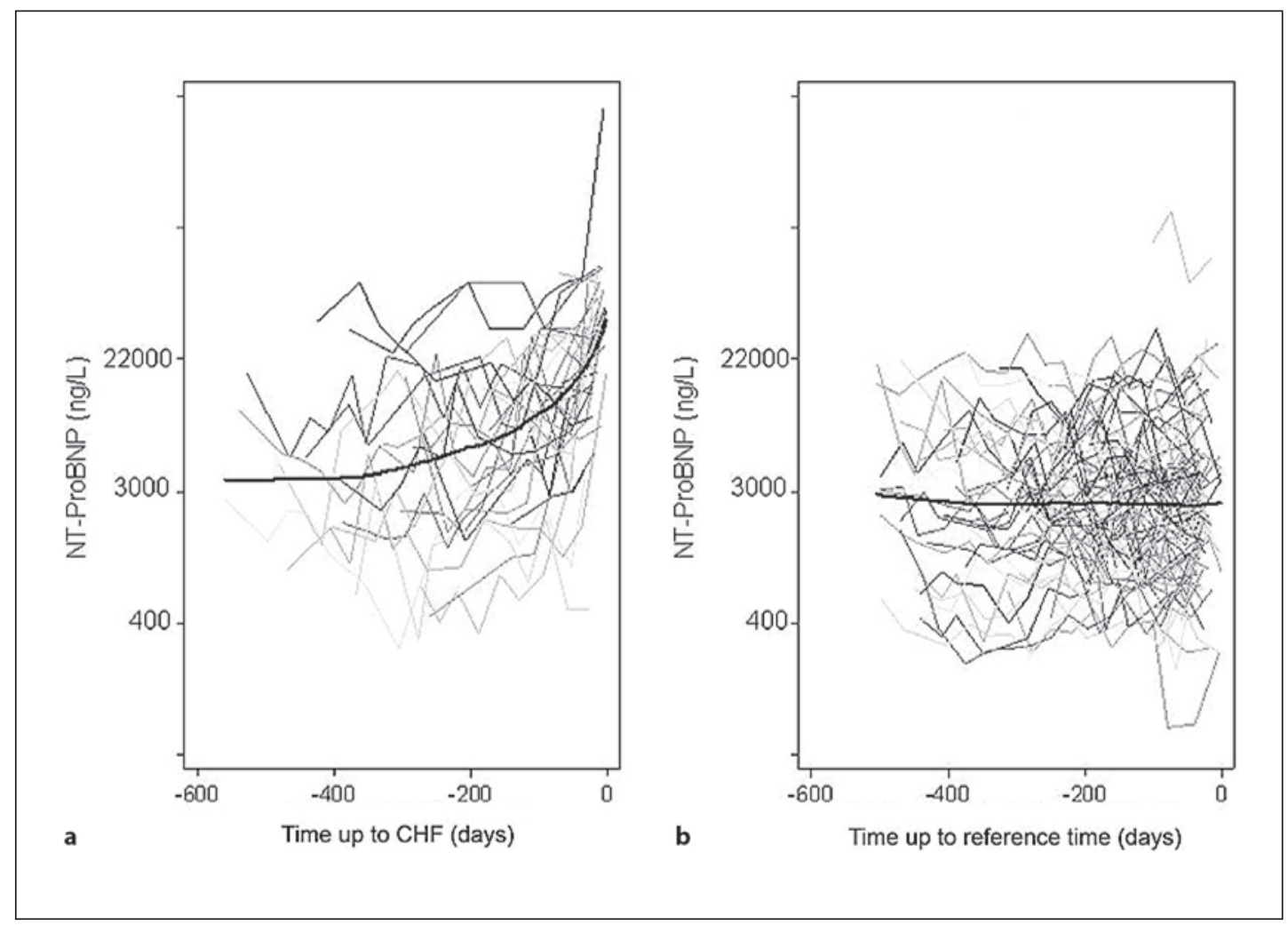

Fig. 1. Monthly monitoring of NT-proBNP in HD patients. The evolution of NT-proBNP measurements before a CHF event (a) compared to the evolution of NT-proBNP measurements at the same time for controls (b) in a nested case control study.

Fig. 2. Performance of NTproBNP as prognostic marker for $\mathrm{CHF}$ in HD patients. ROC curves for the relative change in the NT-proBNP plasma level at two consecutive visits as a predictor of a CHF event.

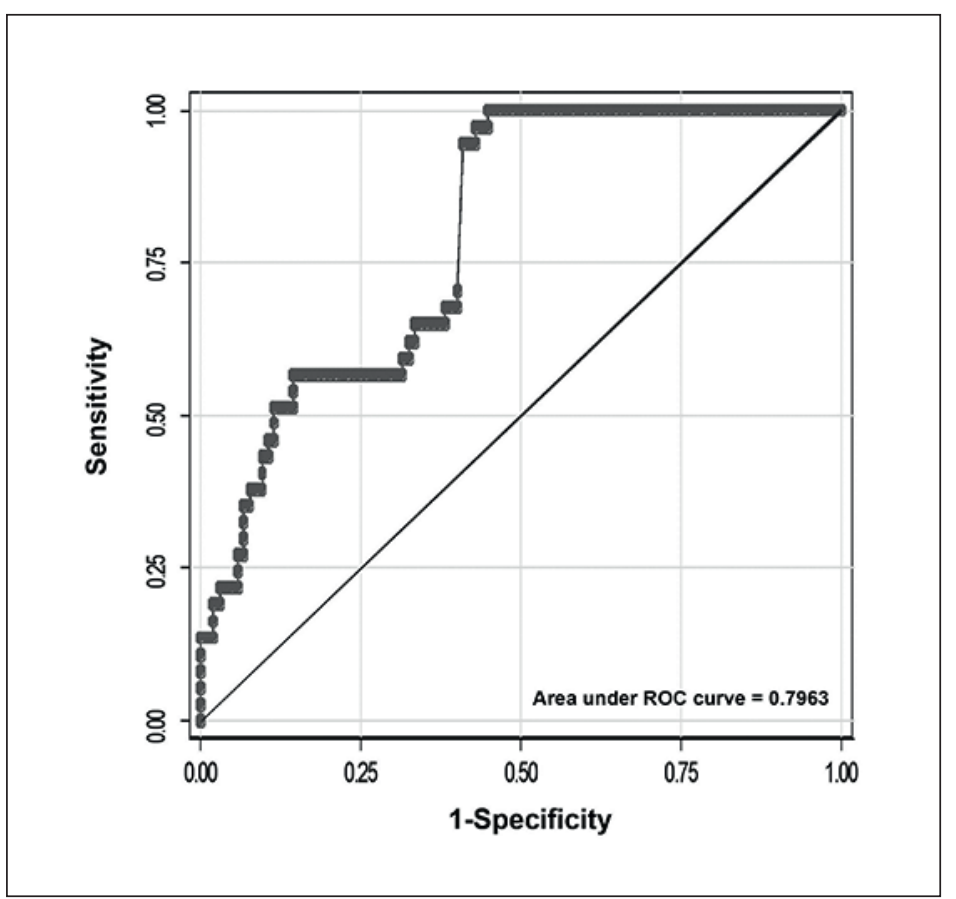


proBNP, the sensitivity of the test was 0.57 and the specificity 0.77 . If the threshold was chosen in favour of sensitivity, then, if all positive relative changes were considered as predictors, the sensitivity was 0.97 but at the price of a low specificity of 0.59 . Only 1 patient had a decrease in NT-proBNP between the two last visits before a CHF event.

\section{Discussion}

These data show that the relative change in NT-proBNP plasma levels is a significant risk predictor of a CHF event. All patients were monitored by monthly NT-proBNP plasma level measurements. A relative increase of $20 \%$ in NT-proBNP measurements was associated with a risk of developing a CHF event during the next month, with a sensitivity of 0.57 and a specificity of 0.77 , given by the ROC curve analysis.

Of note, baseline NT-proBNP levels were higher among patients who suffered a CHF event than among those who did not. Wang et al. [11] made similar observations and concluded that baseline NT-proBNP measurement was an important risk predictor of cardiovascular congestion in peritoneal dialysis patients; the baseline NT-proBNP level was noted to be at least 3-fold higher among patients who developed subsequent circulatory congestion.

However, the present data on monitoring serial NT-proBNP measurements provide more important prognostic information that could potentially be used for assessing treatment adequacy and adjusting therapy in the HD population. Physicians should be alerted when NT-proBNP measurements are increasing. Indeed, if all positive relative changes were considered as predictors of a CHF event, the sensitivity would be high and the specificity of the test by ROC analysis would be 0.59 . Although the specificity is not so high, we know that inflammation, malnutrition, and anaemia are significantly related to NT-proBNP levels.

Gutierrez et al. [8] measured baseline NT-proBNP concentrations in incident HD patients and calculated the change in concentrations after 3 months in a subset of 585 patients. The longitudinal changes in NT-proBNP concentrations were associated with subsequent mortality. Patients with the greatest increase in NT-proBNP after 3 months of dialysis had a 2.4-fold higher risk of mortality than those with the greatest decrease in NT-proBNP. Interestingly, a change in weight was only weakly associated with changes in NT-proBNP in the univariate analysis, and this association was attenuated in the multivariate-adjusted analysis. These data suggest that monitoring NT-proBNP not only reflects fluid volume overload but also myocardial damage.

An increased BNP level is a sensitive marker of LV diastolic dysfunction in HD patients. Thus, in addition to monitor NT-proBNP levels, other methods assessing dry weight should be performed, including clinical examination, bioimpedance analysis, and relative plasma volume monitoring [12]. Recently, Westenbrink et al. [13] have reported that BNP and interdialytic fluid retention are independent and incremental predictors of mortality in HD patients.

Dry weight is a very subjective parameter based first on clinical examination and blood pressure. Patients with altered LV function suffer from anorexia, and, in our study, the body mass index was lower in the group of patients who subsequently developed a CHF event. For these patients, it could be difficult to determine the clinically overload status and the risk of CHF event.

Whereas there is growing enthusiasm for longitudinally following natriuretic peptide concentrations in patients with cardiac failure to help guide therapy [14-18], it is unclear whether employing a similar strategy would be advantageous in HD patients [19]. Prospective randomised studies are needed to determine the cost analysis and cost-effectiveness of NT-proBNP-guided therapy in the HD population. 


\section{Conclusion}

A dynamic change in the NT-proBNP plasma level is an important risk predictor of CHF events. We showed an increase of NT-proBNP concentrations before the occurrence of a CHF event in HD patients. Prospective randomised studies are needed to determine whether specific therapeutic interventions driven by longitudinal changes in NT-proBNP can significantly improve outcomes in HD patients.

\section{Acknowledgements}

The authors want to thank Ms. Claudine Abahouni and the ABC Laboratory's staff for biochemical analysis and technical support.

\section{Disclosure Statement}

The authors have no conflict of interest to declare.

\section{References}

-1 Agarwal R: Hypervolemia is associated with increased mortality among hemodialysis patients. Hypertension 2010;56:512-517.

-2 Januzzi JL Jr, Camargo CA, Anwaruddin S, Baggish AL, Chen AA, Krauser DG, Tung R, Cameron R, Nagurney JT, Chae CU, Lloyd-Jones DM, Brown DF, Foran-Melanson S, Sluss PM, Lee-Lewandrowski E, Lewandrowski KB: The N-terminal Pro-BNP investigation of dyspnea in the emergency department (PRIDE) study. Am J Cardiol 2005;95:948-954.

-3 Braunwald E: Biomarkers in heart failure. N Engl J Med 2008;358:2148-2159.

4 Wang AY, Lai KN: Use of cardiac biomarkers in end-stage renal disease. J Am Soc Nephrol 2008;19: $1643-1652$.

-5 Locatelli F, Vigano S: Are natriuretic peptides a reliable marker for mortality in ESRD patients? Nephrol Dial Transplant 2010;25:347-349.

-6 Satyan S, Light RP, Agarwal R: Relationships of N-terminal pro-B-natriuretic peptide and cardiac troponin $\mathrm{T}$ to left ventricular mass and function and mortality in asymptomatic hemodialysis patients. Am J Kidney Dis 2007;50:1009-1019.

-7 Zoccali C, Mallamaci F, Benedetto FA, Tripepi G, Parlongo S, Cataliotti A, Cutrupi S, Giacone G, Bellanuova I, Cottini E, Malatino LS: Cardiac natriuretic peptides are related to left ventricular mass and function and predict mortality in dialysis patients. J Am Soc Nephrol 2001;12:1508-1515.

-8 Gutierrez OM, Tamez H, Bhan I, Zazra J, Tonelli M, Wolf M, Januzzi JL, Chang Y, Thadhani R: Nterminal pro-B-type natriuretic peptide (NT-proBNP) concentrations in hemodialysis patients: prognostic value of baseline and follow-up measurements. Clin Chem 2008;54:1339-1348.

-9 Troughton RW, Frampton CM, Yandle TG, Espiner EA, Nicholls MG, Richards AM: Treatment of heart failure guided by plasma aminoterminal brain natriuretic peptide (N-BNP) concentrations. Lancet 2000;355:1126-1130.

-10 Clerico A, Recchia FA, Passino C, Emdin M: Cardiac endocrine function is an essential component of the homeostatic regulation network: physiological and clinical implications. Am J Physiol Heart Circ Physiol 2006;290:H17-H29.

-11 Wang AY, Lam CW, Yu CM, Wang M, Chan IH, Zhang Y, Lui SF, Sanderson JE: N-terminal pro-brain natriuretic peptide: an independent risk predictor of cardiovascular congestion, mortality, and adverse cardiovascular outcomes in chronic peritoneal dialysis patients. J Am Soc Nephrol 2007;18: 321-330. 
-12 Sinha AD, Light RP, Agarwal R: Relative plasma volume monitoring during hemodialysis aids the assessment of dry weight. Hypertension 2010;55:305-311.

-13 Westenbrink BD, Hovinga TK, Kloppenburg WD, Veeger NJ, Janssen WM: B-type natriuretic peptide and interdialytic fluid retention are independent and incremental predictors of mortality in hemodialysis patients. Clin Nephrol 2011;76:373-379.

- 14 Jourdain P, Jondeau G, Funck F, Gueffet P, Le Helloco A, Donal E, Aupetit JF, Aumont MC, Galinier M, Eicher JC, Cohen-Solal A, Juilliere Y: Plasma brain natriuretic peptide-guided therapy to improve outcome in heart failure: the STARS-BNP Multicenter Study. J Am Coll Cardiol 2007;49:1733-1739.

-15 Adlbrecht C, Huelsmann M, Berger R, Moertl D, Strunk G, Oesterle A, Ahmadi R, Szucs T, Pacher R: Cost analysis and cost-effectiveness of NT-proBNP-guided heart failure specialist care in addition to home-based nurse care. Eur J Clin Invest 2011;41:315-322.

-16 Kim HN, Januzzi JL Jr: Biomarkers in the management of heart failure. Curr Treat Options Cardiovasc Med 2010;12:519-531.

17 Palazzuoli A, Antonelli G, Quatrini I, Nuti R: Natriuretic peptides in heart failure: where we are, where we are going. Intern Emerg Med 2011;6:63-68.

- 18 Dhaliwal AS, Deswal A, Pritchett A, Aguilar D, Kar B, Souchek J, Bozkurt B: Reduction in BNP levels with treatment of decompensated heart failure and future clinical events. J Card Fail 2009;15: 293-299.

-19 Troughton RW, Lewis LK, Yandle TG, Pemberton CJ, Nicholls MG: B-type natriuretic peptides: looking to the future. Ann Med 2011;43:188-197. 